\title{
Understanding Artificial Intelligence
}

Civilization advances by extending the number of important operations we can perform without thinking about them.

- Alfred North Whitehead

\section{IN THE BEGINNING}

The concept of AI has ancient origins. Around the eighth century BCE, the Greek poet Homer wrote in the Iliad about Hephaestus, the god of fire and a skilled inventor. Hephaestus built golden automata, or self-operating machines, to help him work. Not only did Hephaestus build attendants for himself with "intelligence in their hearts" and the "appearance [of] living young women,"1 he also built autonomous vehicles that could travel to and from the home of the gods and a lethal autonomous weapon system named Talos that patrolled the beaches of Crete. By contrast, Amazon's assistant Alexa, Tesla's Autopilot system, and the Russian Federation's military robot FEDOR seem quaint, although Homer was vague on how mere mortals could enable such constructs.

Stories of artificial beings endowed with humanlike intelligence have pervaded countless histories and cultures. The most famous golem narrative in the Jewish tradition involves Judah ben Loew, the late sixteenth-century chief rabbi of Prague, who is said to have fashioned a humanlike figure from clay and brought it to life with rituals and incantations. This golem helped defend against anti-Semitic attacks and pogroms, and it even performed household chores. There are many versions of the story. But much like the enchanted broom in Goethe's "The Sorcerer's Apprentice," the golem experiment does not end well - often with a murderous rampage. Creators faring poorly is a recurrent theme in such origin stories. Prometheus, who created humankind in Greek mythology and passed along the technology of the gods, was condemned to suffer eternal torture. Mary Shelley's 1818 story of Frankenstein was about a scientist dedicated to making artificial life, only to later reject his creation. The "monster" ultimately torments and destroys its maker. 
Not all historical AI was myth or fiction; early automata could be quite sophisticated. One of the great Roman engineers, Hero of Alexandria, wrote a treatise called "Pneumatica" that describes how to build numerous machines powered by air, steam, and water pressure. This text was used by engineers until early modern times. Hellenic Egypt had statutes of gods that could speak, move, and even prophesize (although their predictive value is unknown). Mechanists in ancient Greece constructed vending machines, water mills, and perhaps even the world's first mechanical computer used to predict astrological movements - the Antikythera mechanism.

Our incomplete historical record suggests the Antikythera mechanism may have been one of many ancient computers - at least mechanical computers. Originally, the term "computer" was used to refer to a person who manually performed mathematical calculations. Human computers were once critical to navigation, science, and engineering, but they left much to be desired. People as computers were slow and error prone, which could be a fatal flaw when bad math ran a ship aground. In the early nineteenth century, apparently while watching human computers at work, Charles Babbage decided to automate the process. Perhaps at some earlier point a Greek machinist had a similar insight observing human astrologers, which led to the invention of the Antikythera mechanism. Astrological calculations were as important to the ancient Greeks as navigational calculations were to the Victorian English. Ironically, the Antikythera mechanism was found at the bottom of the sea in 1902.

But, back to Babbage. After deciding to create a mechanical computer, Babbage began by designing an automatic calculator he called the "difference engine." This machine would have created tables of values by finding the common difference between terms in a sequence, and in turn this could have been used to generate logarithmic tables and trigonometric functions as well as simple price lists for merchants. Babbage completed various designs but never the actual machine. At that time, its building would have been a herculean task - the machine would have weighed about fifteen tons and contained around 25,000 parts. Other obstacles included the fact that Babbage was perpetually running out of funds and getting distracted by side projects, which included campaigning against street noise and perfecting an "infallible" system for gambling on horse racing that proved fallible.

While the difference engine may have been the first modern automatic calculator, its most important contribution may have been to inspire Babbage with a grander distraction: the analytical engine, the first general-purpose computer. Unlike earlier machines, the analytical engine could have carried out a wide range of operations using programs contained on punch cards. Ada Lovelace, the English mathematician and writer who sponsored Babbage, developed an algorithm that would have allowed the machine to generate a sequence of Bernoulli numbers, 
which have an important role in mathematics, essentially making her the first computer programmer. Lovelace may have been the first to recognize the machine's potential beyond pure calculation.

Babbage continued to design various iterations of the analytical engine until his death in 1871, but he never actually built the computer. Babbage's inability to complete his work, combined with his anger at the British government for what he believed was inadequate support, left him embittered and disappointed at his end. He was appreciated - although controversial - in his time, but the true extent of his genius was not recognized until later scientists realized that the analytical engine had anticipated almost every aspect of present-day computers. Today, Babbage is considered the "father of computing," even though the first fully functional generalpurpose computer, the Electronic Numerical Integrator and Calculator (ENIAC), was not completed until 1946. ENIAC weighed 30 tons and took up around 1,800 square feet. While scant comfort to Babbage, after his death two versions of the difference engine were successfully constructed and operated, and efforts are currently underway to build his analytical engine.

Hundreds of years before Babbage, Leonardo Da Vinci constructed what may have been the first humanoid robot in Western civilization - Leonardo's mechanical knight. It could sit, stand, raise its visor, and maneuver its arms by means of a series of pulleys and cables. Leonardo created a "robot" before the term existed. The word was coined in a 1920 play R.U.R. (an acronym for Rossum's Universal Robots) by playwright Karel Capek, who based it on the Czech word for "forced labor." The play takes place around the year 2000 in a world where robots are cheap, ubiquitous, and indispensable. These factory-built constructs are capable of independent thought and are creatures of flesh and blood rather than gears and pulleys. Alas, the robots' creators fare about as well as the golem. In Capek's play, the robots initiate a rebellion that more or less exterminates humanity.

Even early in the twentieth century, long before modern computers made the visions of The Terminator and The Matrix appear plausible, the themes in R.U.R. had widespread appeal. By 1923, the play had been translated into thirty languages, and the word "robot" had entered the English lexicon. Killer robots and the AI apocalypse continued as recurring themes after R.U.R. One of the most famous science fiction writers of the twentieth century, Isaac Asimov, wrote extensively about a future filled with AI. He proposes a set of ethical rules he calls the Three Laws of Robotics, which are hardwired into the "positronic brains" of robots and prevent them from turning against their creators:

(1) A robot may not injure a human being or, through inaction, allow a human being to come to harm.

(2) A robot must obey orders given it by human beings except where such orders would conflict with the First Law. 
(3) A robot must protect its own existence as long as such protection does not conflict with the First or Second Law. ${ }^{2}$

Many of Asimov's stories explore how these deceptively simple rules can result in unexpected and sometimes destructive outcomes. Sometimes, both action and inaction result in harm, and robots may need to cause a lesser harm to prevent a greater one. In Asimov's later stories, some robots come to understand that people, left to their own devices, are perpetually harming themselves and others and that the best way to prevent this harm is for robots to rule over humanity.

\section{The Birth of Modern AI}

In 1956, a decade after ENIAC, the term "artificial intelligence" was coined by computer scientist John McCarthy. He organized the Dartmouth Artificial Intelligence conference, which is often credited with establishing AI as a research discipline. In his proposal for the event, McCarthy defines AI as follows: "For the present purpose the artificial intelligence problem is taken to be that of making a machine behave in ways that would be called intelligent if a human were so behaving." 3 The conference proceeded "on the basis of the conjecture that every aspect of learning or any other feature of intelligence can in principle be so precisely described that a machine can be made to simulate it."

Since the 1956 conference, AI as both a research discipline and practical technology has seen its share of ups and downs. In the 1950s and 196os, optimism prevailed that machines would soon exhibit, and exceed, human levels of intelligence. Computers developed the capabilities to solve algebraic problems, competitively play games like checkers, and speak English. In the 1970s, that optimism faded when promised results failed to materialize and funding dried up. This downturn came to be known as the First AI Winter. It ended in the 1980s as "expert system"-based AI, which solves problems using logical rules derived from the knowledge of specialists, enjoyed some significant successes. But in the 1990s, a sense again emerged that the capabilities of AI had been oversold, and another period of decreased interest and funding took hold - the Second AI Winter. Some researchers during this time even took to calling their work "machine intelligence," "informatics," or "knowledgebased systems" to avoid association with AI.

Those AI winters have passed, but plenty of hype - another recurring feature of the AI narrative - remains. Hundreds of years before Deep Blue defeated Gary Kasparov at chess, Wolfgang Von Kempelen created the world's first chess-playing machine, the Turk. After showcasing the Turk to Austria-Hungarian Empress Maria Theresa, the machine became a European sensation, performing for, and perhaps defeating, the likes of Napoleon Bonaparte, Benjamin Franklin, and Charles Babbage. The Turk was an ingenious feat of engineering, but only for the machine's ability to 
secretly house a human player. Eventually, the Turk was discredited, then consigned to storage where it was later destroyed in a fire.

Some overhyped automatons have had even less happy endings. In the early 1930s, an apparently autonomous vehicle called the "Phantom Auto" toured the country and amazed the public. The tour ended abruptly in 1932 when the vehicle injured ten pedestrians. The Phantom Auto turned out to be a remotely operated Ford Tin Lizzy, and its operators were arrested. Today, experts remain divided on whether AI has finally reached a point of runaway progress or another winter is coming. Some thought leaders, such as Ray Kurzweil, one of Google's directors of engineering, predict computers will have human levels of intelligence in about a decade. On the other hand, in 2018 a robot touted as the most advanced machine ever created in Russia turned out to be a man in a suit. ${ }^{4}$

\section{DEFINING AI}

Intelligence is whatever hasn't been done yet.

- Larry Tesler

More than sixty years after the term was introduced, AI still has no well-accepted definition. This book will define AI as: an algorithm or machine capable of completing tasks that would otherwise require cognition. Cognition refers to mental capabilities and the process of acquiring knowledge and understanding through thought. This is a deliberately broad definition of AI that focuses on what it does rather than how it is designed. Perhaps the most popular textbook on the subject defines $\mathrm{AI}$ as "the designing and building of intelligent agents that receive percepts from the environment and take actions that affect that environment."5 Many modern AI definitions retain McCarthy's original functional emphasis (and circular logic) - a machine that completes tasks traditionally requiring human "intelligence."

Defining "AI" begs definitions of its two linguistic components: "artificial" and "intelligence." Artificial seems more straightforward - something made by people rather than nature. Today, at least, it is possible to distinguish natural from artificial, even if the distinction shows signs of blurring. Researchers have created biological computers that use natural proteins and DNA to perform calculations involving the storing, retrieving, and processing of data. As this technology develops, it may be possible to engineer biological systems that have the functional capabilities of mechanical computers. Not content with making machines out of natural building blocks, researchers are also hard at work trying to upgrade people. Genetically modified "designer" babies have already been created using technologies like CRISPR, mitochondrial replacement, and in vitro gametogenesis. Elon Musk has launched Neuralink, a venture to develop a brain-computer interface so that people can be 
more competitive with AI. While that technology is still in its infancy, people are already being implanted with an increasingly sophisticated range of medical devices.

Intelligence is harder to define or at least make sense of in the context of AI. Shane Legg and Marcus Hutter surveyed a number of prominent informal definitions of intelligence, and they argue that intelligence is commonly defined in terms of "an agent's ability to achieve goals in a wide range of environments." ${ }^{6}$ Intelligence is a feature commonly associated with people, sometimes even considered their defining characteristic. More than two thousand years ago, the philosopher Aristotle essentially argued that human intellect - the capacity to make rational decisions - separates people from other animals. Modern philosophers (and translators) are still arguing about what he really meant.

The philosopher René Descartes believed people are guided by an immaterial, or spiritual, mind while the rest of nature, including animals, is nothing more than a series of mindless objects driven inexorably by the laws of physics. He thus thought it would be possible to build an automaton indistinguishable from an animal but not from a person. A machine, he argued, can never use words or put together signs in the way we "declare our thoughts to others." Even if it could give a poor imitation of speech, it could certainly not give an "appropriately meaningful answer to what is said in its presence, as the dullest of men can do." ${ }^{8}$ Descartes does not appeal to ethereal qualities like a soul or emotion to distinguish people from machines and animals but to communication and reasoning abilities already achieved by today's AI.

People are generally assumed to be smarter than other animals, but when individuals lack intelligence they are not considered less human, and other animals are capable of some fairly intelligent behavior. Chimpanzees can outperform some human players at some speed and memory-based games, octopuses use tools and socialize, dolphins have unique names and sophisticated means of communication, and elephants exhibit empathy. Koko the gorilla famously learned a modified version of American Sign Language, understood around 2,000 English words in addition to the signs, and named and adopted a kitten. She passed away in 2018. But in her youth, she scored between 70 and 90 on intelligence quotient (IQ) tests. A significant number of people score in Koko's range, and a score of 100 is the median. There is no reason Koko and a person of Koko-like knowledge and sophistication should not both qualify as intelligent. Charles Darwin similarly thought human intelligence is not different from other animal intelligence in kind, merely in degree.

Whether a machine with similar capabilities should qualify as intelligent is more controversial. People have attempted to subject AI to modified IQ tests, and a study in 2013 resulted in press coverage that claimed the smartest computers were as intelligent as four-year-old children. The study showed AI's performing relatively well on the verbal portion of an IQ test and poorly on the comprehension portion. 
While AI should be good at memorizing words, an excellent vocabulary combined with poor comprehension does not equal a child with common sense. Comparing people and machines along a single dimension of intelligence has limited meaning. Google Translate can interpret more than a hundred languages almost instantly, and while faster and more versatile at translation than any imaginable person, it cannot write music, play poker, or have an existential crisis. AI like Google Translate is clearly different in kind from human intelligence.

In 1904, the psychologist Charles Spearman advanced the concept of "general intelligence." He argues that people have a single general intelligence that determines cognitive performance in addition to narrow task-specific abilities. He was aware that people perform better at some tasks than others but found that people who do well in one area tend to do well in many domains. Someone good at math is more likely to have a strong vocabulary than someone with poor math skills. Modern advocates of human general intelligence are more likely to subscribe to the belief that IQ scores are a valid measure of intelligence. Critics argue that the concept of general intelligence is not supported by evidence and devalues other important abilities.

Today's machines lack general intelligence - all modern AI is narrow or specific. It focuses on discrete problems or works in specific domains. For instance, "Watson for Genomics" can analyze a genome and provide a treatment plan, and "Chef Watson" can develop new food recipes by combining existing ingredients. However, Watson for Genomics cannot respond to open-ended patient queries about their symptoms nor can Chef Watson run a kitchen. New capabilities could be added to Watson to do these things, but Watson can only solve problems it has been programmed to solve.

By contrast, one of the earliest goals of AI development has been the creation of general AI that would be able to successfully perform any intellectual task a person could. General AI could even be set to the task of self-improvement, resulting in a continuously improving system that surpasses human intelligence - what philosopher Nick Bostrom terms artificial superintelligence. Artificial superintelligence could then innovate in all areas, resulting in progress at an incomprehensible rate. Such an outcome has also been referred to as the intelligence explosion or the technological singularity. This idea has been popularized in recent years, but it is an old concept. The mathematician Irving John Good wrote in 1965, "The first ultraintelligent machine is the last invention that man need ever make." 9

Experts are greatly divided on when, and if, general AI will be developed. Many industry leaders predict based on historical trends that general AI will exist within the next couple of decades. Others believe the magnitude of the challenge has been underestimated, and that general AI will not be developed in the twenty-first century. While there are conflicting predictions, in 2013 hundreds of AI experts were surveyed on their predictions for artificial general intelligence development. ${ }^{10}$ On average, participants predicted a 10 percent likelihood that general AI would 
exist by 2022 , a 50 percent by 2040 , and a 90 percent by 2075 . In a similar survey, 42 and 25 percent of participants said general AI would happen by 2030 and 2050 , respectively. ${ }^{11}$ In addition, 10 percent of participants reported they believed superintelligent AI would develop within two years of general artificial intelligence, and 75 percent predicted this would occur within thirty years.

\section{CAN AI THINK?}

Recently there has been a good deal of news about strange giant machines that can handle information with vast speed and skill ... . These machines are similar to what a brain would be if it were made of hardware and wire instead of flesh and nerves .... A machine can handle information; it can calculate, conclude, and choose; it can perform reasonable operations with information. A machine, therefore, can think.

$$
\text { - Edmund Berkeley (1949) }
$$

In 1950, Alan Turing, the computer scientist who launched and inspired much of AI, published a paper in which he "propose[s] to consider the question, 'Can machines think?"'12 He proceeds to dismiss inquiries about whether a machine understands what it is doing and reframe the question to ask whether a computer could functionally imitate a person. Turing then argues that a machine would functionally think if it could pass an "imitation game" in which a computer would have to successfully pretend to be a person to a suspicious judge. Turing predicted computers would have enough storage capacity to pass what has subsequently been named the Turing test in about fifty years, that is, by the year 2000 .

Another school of thought about AI says that intelligence requires understanding. In this view, machines do not qualify as intelligent regardless of what they can do because they do not comprehend what they are doing. Action without understanding merely simulates intelligence, even for a superintelligent AI. Instead of specific and general AI, philosopher John Searle puts the distinction between acting and understanding in terms of strong (thinking) and weak (unaware) AI.

To illustrate, consider Searle's Chinese room thought experiment. Assume a person has no Chinese language proficiency and is kept in a room where a message in Chinese characters is presented to her through a small opening in the wall by a native Chinese speaker. She cannot understand the meaning of the characters, but she has a book that contains every possible combination of characters, together with a corresponding appropriate response. She looks them up in the book, transcribes the appropriate response onto a piece of paper (assume she can figure out how to write Chinese characters), and passes her response back. The person who sent her the initial message does receive an appropriate response.

Functionally, she would be mimicking communication like a native speaker of Chinese, albeit very slowly. However, she would not comprehend the meaning of the message she received or her response. This is a model for thinking about AI. If 
Google Translate is substituted for the Chinese room, there is a faster, and less accurate, version of the same phenomenon. Google Translate may pass the Turing test, but it is weak AI - it lacks semantic understanding.

Part of the challenge with arguing machines cannot think is that human thought remains incompletely understood. For generations, there was little empirical evidence about the nature of thought, which was the domain of philosophers, theologians, and poets who could endlessly debate the subject in the absence of definitive proof. Eventually, neurobiologists wedged their way into the debate, some of whom argue that thought is a physical phenomenon that inevitably results from biological systems like a person's higher nervous system. If so, a living being similar to a person, if not a dolphin or a primate, would experience something close to human consciousness. For that matter, if the mind is no more than the sum of a biological system, there is no reason why it should not be possible to re-create the same phenomenon with a machine - or for a machine to improve upon human thought.

In this view, a person is simply a machine, albeit a phenomenally complex one a "meat machine," as cognitive scientist Martin Minsky eloquently puts it. This mind-machine view conflicts with the beliefs of a long line of philosophers reaching back to Plato who believed that the human mind or soul is distinct from the physical body. Descartes similarly thought that the mind and body are distinct in substance and nature and that while the body can be divided, the mind is indivisible, like the concept of a soul in Christian theology.

More recently, philosopher David Chalmers has weighed in on the mind-body issue to distinguish between the easy and hard problems of consciousness. The easy problems include explaining the ability to focus attention, discriminate, integrate information, and so forth. These are problems with mechanistic solutions, and neurobiologists and computer scientists are quickly making progress toward understanding the approach of the human nervous system and developing comparable AI capabilities. The hard problem of consciousness refers to why people have phenomenal experiences, which is to say the sensation of internal states. Mechanistic explanations seem unable to explain what it is like to see red, hear music, or feel cold. The existence of phenomenal consciousness in a material world remains a metaphysical puzzle.

We are far from fully understanding consciousness, but there are things we do understand. For instance, consciousness can be localized, at least to a degree, within the central nervous system. The central nervous system is composed of the brain and spinal cord, and the brain has three major parts. The cerebrum, consisting of a right and left hemisphere, is the largest and most evolved part of the brain and is responsible for higher functions like speech, reasoning, learning, and hearing; the cerebellum coordinates muscle movements and maintains posture and balance; and the brainstem connects the cerebrum and cerebellum to the spinal cord and regulates things like body temperature, breathing, and heart rate. People who have lost their spinal cords and even suffered massive injuries to their cerebellums and 
brainstems can still retain normal consciousness. This suggests that consciousness is generated in the cerebrum. Consciousness can even be localized further within the cerebrum. People who have had large portions of their prefrontal cortex (the part of the cerebrum nearest the forehead) removed or destroyed often do not experience any effect on their conscious experience.

However, the removal of even small parts of the posterior cortex can result in dramatic losses of conscious content - the inability to see or recognize motion, color, or space. The cerebrum has a very different neural architecture than the cerebellum and has a relatively less dense concentration of neurons that are highly connected in networks and activate in complex feedback loops. By contrast, the cerebellum has a higher concentration of neurons but in relatively uniform and parallel structures where groups of neurons function independently in a feed-forward circuit (one set of neurons activates the next). If a person is merely a biological machine, then the cerebrum's structure is likely a key part of the consciousness puzzle, or even the key to future machines with self-awareness.

But what has all this done to resolve the question of whether AI thinks? On one level the question can be addressed by noting that AI does not think the way a person does. AI is not conscious or self-aware in the same sense a person is. On another level, the question can be addressed by noting that it remains more relevant to philosophers than to policymakers. The reason for this is that the social benefits of AI are based on what AI can functionally accomplish, regardless of how it processes information. AI does not need to mimic human thought processes or perceive the meaning of its actions. It simply needs to automate human intellectual activity. AI does not need to think - just do.

\section{AI TYPES AND APPLICATIONS}

An algorithm is a set of mathematical instructions or rules, which in the form of a software program can instruct computer hardware, the physical components of a machine, to perform specific tasks. Each program is essentially a step-by-step instruction guide, like a cooking recipe, that provides a set of ordered operations to the computer. Hardware runs or executes algorithms, though an algorithm developed and readable by a person is rarely directly usable by a computer. An algorithm is usually written in a high-level programming language (source code) because these are close to natural language. The source code is then usually translated to machine language (object code), which consists of binary values (os and 1s) that provide processor instructions that change a computer's state. To execute an algorithm, a computer needs instructions in a machine language and a hardware processor such as a central processing unit. For example, an instruction may change a stored value somewhere in memory or ask the operating system to print text to a monitor. An analogy has been drawn between software and hardware on the one hand, and mind and body on the other. 
Modern computers have billions of transistors, which can be used as electronic switches. They exist, like binary code, in either an "on" (1) or "off" (o) state. An algorithm can accomplish a task as simple as flipping a single switch, which corresponds to changing one bit of information, and can engage in simple logical reasoning by changing transistors in response to other transistors: "If transistor $\mathrm{X}$ turns on, then transistors $\mathrm{Y}$ and $\mathrm{Z}$ turn on." Complex logical reasoning involves an algorithm's combining multiple operations and can require flipping switches billions of times per second, the acts of which become responsible for everything a computer can do, from translating English to Zulu, modeling a new chemical, or playing a game of backgammon.

There are various techniques, systems, and methodologies for programming algorithms to behave intelligently. Some of these are based on specific goals (e.g., robotics or machine learning) or tools (e.g., logic or neural networks), but philosophical differences and conflicts between institutions and researchers have also influenced AI development. Two of the major AI models are symbolic/classical, what philosopher John Haugeland calls Good Old-Fashioned AI (GOFAI), which encodes a model of a problem and processes input data according to the model to provide a solution, and connectionist systems such as artificial neural networks in which users do not explicitly program rules but allow the AI to discover rules from training data.

\section{Symbolic/Classical AI}

Programming symbolic or classical AI is about creating rules for software to follow. If a specific event occurs, then a particular action will be performed. Symbolic AI has accomplished great feats, such as Arthur Samuel's checkers-playing program in the late 1950s, which learned to beat its programmer and hinted that computers would one day develop superhuman performance. In 1972, another symbolic AI called MYCIN was created to advise physicians on identifying infectious diseases and prescribing antibiotics, and it outperformed infectious disease experts in limited circumstances.

Some early AI researchers like Allen Newell and Herbert Simon believed that symbolic rules govern human psychology. In fact, as early as the 1956 Dartmouth conference, Newell and Simon presented an AI system called "Logic Theorist," which simulated human behavior by relying on heuristics. Heuristics refers to rules of thumb that people use to help make decisions, or general methods of solving programs that make searching through an entire space unnecessary. The Logic Theorist attempted to prove a theorem by guessing a solution and then endeavoring to demonstrate the guess was correct. It independently proved some of the theorems in Principia Mathematica, Alfred North Whitehead and Bertrand Russell's foundational mathematics text, and even found an improved proof to one of the its theorems. If logic and consistency are the defining characteristic of human thinking, 
as Aristotle argued, the Logic Theorist should receive a passing grade. Convincing the establishment was another matter, however, as the Journal of Symbolic Logic refused to publish the proof in an article with the Logic Theorist listed as an author. ${ }^{13}$

Without heuristics, problem-solving approaches may rely on brute-force computational methods, such as generating all possible combinations of symbols to prove a mathematical theorem. Newell and Simon referred to this as the British Museum algorithm - a riff on the infinite monkey theorem, which predicts that a room full of monkeys with typewriters will eventually reproduce by random chance all the books in the British Museum. While theoretically possible, picture this scenario: Monkeys are tasked with typing the word "banana" on typewriters with fifty keys. If they press keys at random, and each key has an equal chance of being pressed, the chance that the first six letters pressed spell banana is $(1 / 50)^{6}$ (one in fifteen trillion six hundred twenty-five billion). Thus, the probability of having the monkeys type banana is more than zero, but it would be luck, indeed, to have it happen even if they tried all day, every day, for the rest of their natural lives.

By the time monkeys start trying to re-create the complete works of William Shakespeare without errors, assuming the secret to immortality has been discovered and applied to the monkeys, they would probably be hard at work at the end of the universe. To drive the point home, someone actually performed an experiment in which a computer keyboard was left in an enclosure of six Celebes crested macaques in Paignton Zoo in England. Over the course of a month in 2003, the monkeys produced about five pages of writing that largely consisted of the letter "S."

Thankfully, computers are better than monkeys at this sort of work, yet even the most powerful computers are limited when dealing with sufficiently large numbers. Checkers has fairly simple rules but a staggering number of possible moves - some $10^{34}$ possibilities. It is hard to think about numbers that large, but computer scientist Jonathan Schaeffer compares it to draining the Pacific Ocean and refilling it with an eight-ounce cup, one scoop at a time. Schaeffer spent decades developing an AI called Chinook that eventually solved checkers in 2007. Chess is more complex, having about $10^{120}$ possible board configurations, which puts a solution beyond the limits of feasible technology. Deep Blue, for example, could only generate every possible move for the next eight moves, and both checkers and chess are nothing like Go, which has $10^{172}$ possible board configurations. While brute-force computation can work very well with problems that have a limited number of possible solutions, it is less useful for others.

\section{Connectionist AI}

Connectionist systems generate intelligent behavior by representing rules in interconnected networks of simple and even uniform units such as artificial neurons. Many of the most dramatic advances achieved by AI in recent years have come from improvements in connectionist techniques such as machine 
learning, artificial neural networks, and deep learning. Machine learning allows computers to learn from examples and generate their own rules, which may be more accurate than rules explicitly created by programmers, particularly where the rules are based on large datasets and complex patterns that are difficult for people to directly interpret.

Artificial neural networks, one set of algorithms used in machine learning, are inspired by the architecture of the human brain. Each person's brain has about 90 billion individual neurons, which are interconnected in highly complex networks with perhaps hundreds of trillions of connections. These connections change dynamically, or the strength of them changes, as a result of learning and experience. Similarly, artificial neural networks consist of groups of interconnected layers of algorithms that feed data into each other and that can be trained to complete tasks by altering the relevance of data as they pass between layers. During training, the weights attached to different inputs change until the network produces a desired output, at which point the network has developed the ability to complete a task. Deep learning involves many layers of neural networks, and has been responsible for many of the recent advances in speech recognition and computer vision.

Connectionist AI was a focus of development efforts on-and-off throughout the twentieth century, but its utility has dramatically improved in the past decade. In 2011, Jeff Dean and Andrew Ng built a large neural net across 16,000 of Google's server processors and fed it 10 million unlabeled YouTube images. The network spent three days processing the data and taught itself to recognize human faces, human bodies, and cats with accuracy rates of about 75 to 82 percent. $^{14}$ The same learning method was then able to substantially outperform other $\mathrm{AI}$ systems at a major image recognition contest called the ImageNet Challenge in which programs compete to correctly classify and detect classes of objects and scenes.

This was a breakthrough in machine learning and the start of the Google Brain project. The following year, two graduate students, Alex Krizhevsky and Ilya Sutskever, working at University of Toronto with Geoffrey Hinton built a neural network model called AlexNet, which dominated the ImageNet contest by a margin of more than 10 percent. ${ }^{15}$ Google hired all three researchers. Machine learning now plays a major role in a wide variety of Alphabet and Google technologies. For instance, AlphaGo utilizes neural networks.

\section{Advances in AI and Their Applications}

AI's increased capabilities are largely due to three parallel developments: more advanced software, greater computing power, and growth of big data. These advances are moving - and have moved - quickly, with improvements taking less 
and less time with each AI incarnation. Deep Blue, which awed programmers and laypersons alike in 1997, has been retired, but the machine was ten million times faster than the first machine used to play chess in 1951 and was capable of evaluating more than 200 million moves per second. A Samsung Galaxy Sio smartphone is fifty times faster than Deep Blue. Likewise, the modern iPhone has around 100,000 times more processing power than the Apollo 11 computer that put Neil Armstrong and Buzz Aldrin on the moon in 1969, and it holds millions of times more memory.

In 2017 , the International Data Corporation forecasted that the "global datasphere" will include 163 zettabytes of information by 2025 , which is more than ten times the 16.1 zettabytes of data available in 2016 . It is difficult to conceptualize data on that scale: 163 zettabytes are roughly equivalent to sixteen million years of highdefinition video or twenty billion US Libraries of Congress. Big data are critically important for AI, and vice versa. AI can deal with large and complex datasets in ways that people cannot. Big data also can be used to train some forms of AI to progressively improve their performance without explicit programming. The importance of data for AI and other purposes has led to data's being called the new oil or the oil of the twenty-first century (an occasionally criticized metaphor). As the European Commission noted in 2018, "Data is the raw material for most AI technologies."16

All big data are not created equal, however, and "garbage in, garbage out" is a maxim in the AI community. For big data to have value for AI, the right kinds of data need to be collected, aggregated, and appropriately utilized. Take the example of an AI's analyzing health care insurer electronic health records to determine if a pharmaceutical medication has adverse events. The AI might need data on exposures (with dates), outcomes, and other health conditions together with insurer enrollment and demographic information, and it might be helpful to link datasets across different time points and insurers. Failure to obtain comprehensive and accurate data used for AI input might result in an incorrect AI output, or if the input is used to train an AI, it might result in an inaccurately biased AI.

Even if the necessary data exist, they still need to be brought together and appropriately translated for AI. Most AI systems are unable to work with unstructured data, which are generally distinguished from structured data by their degree of organization. Structured data usually exist in relational databases where they are categorized and sorted into distinct, easily searchable fields. Unstructured data, by contrast, do not have a predefined data model. They may exist as a heterogenous free-form mix of text, audio, video, and social media postings that have not been labeled or sorted. Most big data now are unstructured, and significant cost and effort are needed to structure them.

AI has enjoyed recent advances in a variety of areas such as computer vision, which deals with how machines can gain a high-level understanding from images or videos, essentially automating the human visual system. In 2015, Microsoft announced it had beat the ImageNet Challenge's dedicated human labeler by classifying more than 95 percent of the images correctly. ${ }^{17}$ In 2017 , twenty-nine of the thirty-eight competing teams classified more than 95 percent of them correctly. ${ }^{18}$ These improvements have 
translated to practical gains as well. For instance, Facebook's image recognition technology now identifies people, objects, expressions, activities, events, and spaces. This AI has a very rich source of training data - more than two billion photos are uploaded daily to Facebook and platforms it owns: Instagram, WhatsApp, and Messenger. These are impressive achievements, though machines still struggle with visual identification in many situations that are no challenge to people.

AI has made similar advances in natural-language processing to which anyone who uses programs like Siri, Alexa, or Cortana can attest. The idea of machine translation as well as predictions that fully realized machine translation would exist within a few years has been around since the 1950s. Those predictions were unrealistic, but today's machine translation has come a long way. Google Translate, for instance, supports more than a hundred languages, including many by photo, voice, and even real-time video. The Google Translate app is used by more than 500 million people and translates around 143 billion words daily. ${ }^{19}$

To take another example, Microsoft announced in 2017 that its speech recognition system performed as well as human transcribers in recognizing speech from Switchboard Corpus, a collection of thousands of recorded random conversations. Fifteen years before that, machine translation word-error rates hovered around 20 to 30 percent. In 2017, Microsoft's technology reached an error rate of 5.9 percent, the same as a human transcriber.

AI has already had a substantial economic impact and has the potential to have far more, though it is difficult to compare estimates because they often use different definitions of AI. ${ }^{2 \circ} \mathrm{A}$ study by PricewaterhouseCoopers suggests that AI could contribute $\$ 15.7$ trillion to the global economy in 2030, of which $\$ 6.6$ trillion could come from increased productivity and $\$ 9.1$ trillion from consumption-side effects. ${ }^{21}$ A report by the McKinsey Global Institute claims the disruptions caused by new technologies such as AI will "happ[en] ten times faster and at 300 times the scale, or roughly 3,000 times the impact" of the Industrial Revolution. ${ }^{22}$

\section{AI CHARACTERISTICS}

Understanding AI is important for thinking about how it should be regulated and how it challenges existing legal systems. While a conventional machine could exhibit some or all the following characteristics - directly causing physical changes or behaving with limited predictability or explainability - AI is far more likely to display these characteristics and to a greater extent. A difference in degree along one or several of these axes makes AI worth considering as a distinctive phenomenon.

\section{Limited Explainability}

An AI's actions cannot always be explained. It may be possible to determine what an AI has done but not how or why it acted as it did. For instance, an algorithm may 
decline a job application but be unable to articulate why the application was rejected. This lack of explainability is particularly likely in the case of connectionist AI that learns from data. Even if theoretically possible to explain an AI outcome, it may be impracticable given the complexity of $\mathrm{AI}$, the possible resource-intensive nature of such inquiries, and the need to maintain earlier versions of $\mathrm{AI}$ and specific data.

\section{Specific vs. General and Weak vs. Strong AI}

While AI can already outperform people in breathtaking fashion in some domains, such as board games, in other domains $\mathrm{AI}$ is not that useful. All $\mathrm{AI}$ is designed to perform narrow or specific tasks - not general ones. For instance, DeepMind's AI can beat the world's best human player at Go, but it cannot translate English to French without being programmed to do so. By contrast, the holy grail of computer science research is developing general $\mathrm{AI}$ that can perform any task that a person can perform. But, general and even superintelligent AI are still considered "weak" compared to the self-aware, conscious, and sentient AI that is common in science fiction. This latter sort - "strong" AI - is portrayed as having humanlike abilities to cognitively reason and be morally culpable for its actions. As of today, the prospect of such machines is still safely within the realm of science fiction.

\section{Lack of Predictability}

AI can engage in activities that its original programmers may not have intended. Microsoft's chatbot Tay is sometimes invoked as an example of an AI's acting unpredictably. Tay was touted as an experiment in "conversational understanding," an AI that would get smarter through engagement with people. Soon after the bot's launch on social media sites like Twitter, people barraged Tay with misogynistic, racist, and political vitriol - and Tay responded in kind. For instance, when one user asked, "Is Ricky Gervais an atheist?," Tay replied, "ricky gervais learned totalitarianism from adolf hitler, the inventor of atheism." ${ }^{23}$ Microsoft discontinued Tay within twenty-four hours. While the specific tweet may have been unforeseeable, in retrospect it is surprising that one of the world's largest technology companies would have so little insight into the nature of online discourse. On the other hand, hindsight is $20 / 20$ and a Chinese predecessor of Tay named Xiaoice reportedly engaged in more than forty million conversations without major incident. ${ }^{24}$

\section{Physicality}

Although Tay's activities were entirely digital and limited to the Twittersphere, AI does have the potential to directly cause physical changes when it is embodied in or controls hardware, as in the case of a robot. The first reported robot fatality occurred 
on the fifty-eighth anniversary of R.U.R.'s premiere. In 1979, Robert William, a Ford assembly worker, was killed when a robot's arm struck him in the head. In subsequent litigation, a jury found this accident was due to a lack of safety measures and awarded William's estate $\$ 10$ million in damages. Of course, it is not necessary for AI to control a robot to cause physical change. An entirely digital AI that exists only on the cloud, an interconnected network of remote servers, could stop someone's pacemaker or target a person for unwanted attention by law enforcement.

\section{Autonomy}

Perhaps the most important characteristic of AI from the perspective of AI legal neutrality is its ability to act autonomously - functionally determining for itself how to complete a given task. This characteristic is well illustrated by "The DAO," the most famous attempt to create a decentralized autonomous organization (DAO). The idea was that an entity (organization) would be created on a blockchain, a type of distributed ledger technology (decentralized) used to support cryptocurrencies like Bitcoin. Once set in motion, it would act automatically according to smart contracts (autonomous), which are preprogrammed rules dictating future behavior. In other words, The DAO was an AI that would function independently of its original developers while simultaneously existing across countless computers.

Built by German company Slock.it (and its founders) and launched on the Ethereum blockchain, which generates a cryptocurrency called "Ether," The DAO was meant to operate like a venture capital fund. During a creation period, investors could fund The DAO by purchasing "tokens" using Ether, much like buying shares in a corporation, after which anyone could pitch an investment opportunity that The DAO could fund based on the votes of token holders who, like shareholders in a traditional company, would then receive rewards from profitable projects.

The DAO's code was open-source and made publicly available to anyone to inspect how the program would operate. The primary benefit of this structure is that The DAO's creators would be unable to misappropriate The DAO's funds or misuse the entity. Because it was a technologically cutting-edge project, and it removed the need to trust other people, The DAO was appealing to a particular sort of investor, many of whom were cryptocurrency enthusiasts. In 2016, The DAO resulted in the then-largest crowdfund in history, raising around $\$ 150$ million worth of Ether.

Unfortunately, before any projects could be funded, a hacker exploited a bug in The DAO's programming and started to withdraw its funds. The same "unstoppable" or "unalterable" architecture that investors were attracted to, which prevented The DAO's creators from changing its code for their own benefit, meant they could not fix the faulty code or directly recover the stolen funds. Stopping the hacker required extreme measures from the Ethereum community. A "hard fork" was instituted, which changed the underlying 
blockchain protocol's code and resulted in new and old versions of Ethereum: Ethereum and Ethereum Classic. The new blockchain was maintained by those who supported the intervention, and the legacy blockchain was preserved by those who believed the blockchain should be "immutable" and that contributors to The DAO had only themselves to blame.

In the post-hard fork Ethereum blockchain, users who contributed Ether to The DAO were permitted to withdraw their funds. The hacker, meanwhile, was able to withdraw the funds on the significantly less valuable Ethereum Classic blockchain. The decision to hard fork was controversial in the Ethereum community and significantly reduced the value of the Ethereum blockchain. The hacker was never identified, and no civil or criminal charges were levied against The DAO's creators. The US Securities and Exchange Commission investigated The DAO and reported that its tokens were securities and subject to federal securities laws, which appeared to have been violated.

Ultimately, The DAO did not survive this controversy. However, new DAOs are being created. In 2018, the government of Malta considered legislation that would provide legal personality to DAOs.

\section{Further Thoughts}

AI has already accomplished feats that critics in previous generations considered impossible. If these developments appear unimpressive today, it is only because problems stop seeming so mysterious once they can be solved. Meanwhile, AI with ever-improving capabilities is being integrated into our daily lives and automating activities that once required significant human effort. While difficult to predict the exact trajectory of AI's continued development, it is self-evident that $\mathrm{AI}$ is poised to take over a growing range of activities that were once the exclusive province of people. Al's potential seems virtually unlimited, so the question might not be where $\mathrm{AI}$ is headed but when will it get there. 